R. Ware

Nagoya Math. J.

Vol. 61 (1976), 117-125

\title{
HASSE PRINCIPLES AND THE $u$-INVARIANT OVER FORMALLY REAL FIELDS
}

\author{
ROGER WARE
}

\section{Introduction}

In this paper we investigate the connection between the $u$-invariant, $u(F)$, of a formally real field $F$ as defined by Elman and Lam [2] and certain Hasse Principles studied by Elman, Lam and Prestel in [3].

In section 2 the notion of an effective diagonalization of a quadratic form is introduced and in section 3 it is shown that if $F$ is a field having at most a finite number of orderings such that every form over $F$ has an effective diagonalization (which happens, for example, if $F$ is any field having at most one ordering) then the finiteness of the $u$-invariant is equivalent to the Hasse Principle $H_{n}$ holding for all $n$ larger than some fixed integer $m$.

In section 4 we present two generalizations of a theorem of Kneser which states that if $F$ is a non-formally real field then $u(F) \leq q$, where $q$ denotes the number of distinct square classes of $F$. If $F$ is a formally real field such that every form over $F$ can be effectively diagonalized then it is shown that $u(F) \leq t$ where $t$ is the number of distinct square classes of totally positive elements of $F$ and $H_{n}$ is satisfied for all $n>\frac{1}{2} q$.

I would like to express my thanks to T.Y. Lam who pointed out the improved bounds in the proof of Theorem 3.1 and the statement of Theorem 4.4 .

\section{Notations and terminology}

The terminology and notations will primarily follow [2,3,6]. All fields $F$ will have characteristic different from two, $\dot{F}$ denotes the multiplicative group of $F, \dot{F}^{2}$ the subgroup of non-zero squares, and $\Sigma \dot{F}^{2}$ the

Received September 10, 1975. 
subgroup consisting of all sums of squares (= totally positive elements). Isometries of quadratic forms over $F$ will be written as $\cong, \phi \perp \psi$ and $\phi \otimes \psi$ will denote, respectively, the orthogonal sum and tensor product of two forms $\phi$ and $\psi$, and for any natural number $m$ the form $\phi \perp \phi$ $\perp \cdots \perp \phi$ ( $m$ times) will be denoted by $m \phi$. We will write $\phi=\left\langle a_{1}, a_{2}\right.$, $\left.\cdots, a_{n}\right\rangle$ to mean $\phi$ has an orthogonal basis $e_{1}, e_{2}, \cdots, e_{n}$ with $\phi\left(e_{i}\right)=$ $a_{i} \in \dot{F}$. The Witt ring of non-singular quadratic forms over $F$ will be denoted by $W(F)$ and its torsion subgroup by $W_{t}(F)$. The $u$-invariant of $F$ is defined to be $u(F)=\max \{\operatorname{dim} \phi\}$ where $\phi$ ranges over all anisotropic forms in $W_{t}(F)$ [2].

If $F$ is a formally real field then any ordering $<$ on $F$ induces a ring homomorphism $\sigma_{<}: W(F) \rightarrow Z$ via $\sigma_{<}(\phi)=\sum_{i} \sigma_{<}\left(a_{i}\right)$, where $\phi=\left\langle a_{1}\right.$, $\left.\cdots, a_{n}\right\rangle$ and $\sigma_{<}\left(a_{i}\right)=1$ if $0<a_{i}, \sigma_{<}\left(a_{i}\right)=-1$ if $a_{i}<0$. If $\phi$ is a form over $F, \sigma_{<}(\phi)$ is called the signature of $\phi$ relative to the ordering $<$. From [7, Satz 22] it follows that $W_{t}(F)$ consists precisely of those forms which have signature zero relative to all orderings on $F$. A form $\phi$ is called totally indefinite (or locally isotropic) over $F$ if $\left|\sigma_{<}(\phi)\right|<\operatorname{dim} \phi$ for all orderings $<$ on $F$. Thus a form $\phi$ is totally indefinite if and only if $\phi$ is isotropic over all real closures $F_{<}$of $F$ as $<$ runs through the orderings of $F$. The formally real field $F$ satisfies the Hasse Principle $H_{n}$ (for some $n \geq 2$ ) if every totally indefinite form of dimension $n$ over $F$ is isotropic [3].

We denote by $X=X(F)$ the topological space of orderings on $F$ $[1,5]$. The space $X$ is compact, Hausdorff, and totally disconnected with a subbase of the topology given by the sets $W(a)=\{<$ in $X \mid a<0\}$, $a \in F$. We say $F$ (or $X$ ) satisfies the Strong Approximation Property (SAP) if given any two disjoint closed subsets $U, V$ of $X$ there exists an element $a$ in $F$ which is positive at the orderings in $U$ and negative at the orderings in $V$.

\section{Effective diagonalization of quadratic forms}

A form $\phi=\left\langle a_{1}, a_{2}, \cdots, a_{n}\right\rangle$ over a formally real field $F$ is said to be effectively diagonalized if $W\left(a_{i}\right) \subset W\left(a_{i+1}\right), i=1,2, \cdots, n-1$. The field $F$ is said to satisfy $E D$ if every form over $F$ can be effectively diagonalized.

LEMMA 2.1. Suppose $F$ is a formally real field and $\phi$ is a form which 
can be effectively diagonalized. Then

(i) If $\phi$ is totally indefinite then we can write $\phi=\beta \perp \phi^{\prime}$ where $\beta=\langle a, b\rangle$ is a binary form with a totally positive and $b$ totally negative,

(ii). If $\phi$ is totally indefinite then there exists an integer $m \geq 1$ such that $m \phi$ is isotropic (i.e. $\phi$ is weakly isotropic in the sense of $[3,8]$ ).

(iii) If $\phi \in W_{t}(F)$ then $\phi=\beta_{1} \perp \cdots \perp \beta_{n}$ where $\beta_{i}=\left\langle a_{i}, b_{i}\right\rangle \in W_{t}(F)$ with $a_{i}$ totally positive and $b_{i}$ totally negative. In particular, $\phi$ is strongly balanced in the sense of [7].

(iv) If $\phi \in W_{t}(F)$ with $\operatorname{dim} \phi=2 n$ then $\phi=\phi_{1} \perp \phi_{2}$ with $\operatorname{dim} \phi_{i}=n$, $i=1,2$, and where $\phi_{1}$ has signature $n$ and $\phi_{2}$ has signature $-n$ relative to all orderings on $F$.

Proof. (i ) Write $\phi=\left\langle a_{1}, a_{2}, \cdots, a_{k}\right\rangle$ with $W\left(a_{i}\right) \subset W\left(a_{i+1}\right)$ for all $i$. Since $\phi$ is totally indefinite $W\left(a_{1}\right)$ must be empty and $W\left(a_{k}\right)=X$. Thus $a_{1}$ is totally positive and $a_{k}$ is totally negative so we can take $\beta=\left\langle a_{1}, a_{k}\right\rangle$.

(ii) Write $\phi=\beta \perp \phi^{\prime}$ with $\beta=\langle a, b\rangle \in W_{t}(F)$. Choose $m \geq 1$ so that $m \beta=0$ in $W(F)$. Then $m \phi$ is isotropic.

(iii) Write $\phi=\left\langle a_{1}, a_{2}, \cdots, a_{k}\right\rangle$ with $W\left(a_{i}\right) \subset W\left(a_{i+1}\right)$ for all $i$. Since $F$ is formally real and $\phi \in W_{t}(F)$ it follows that $k=2 n$ is even, $a_{1}, \cdots$, $a_{n}$ are totally positive and $a_{n+1}, \cdots, a_{k}$ are totally negative. Hence we can take $b_{i}=a_{n+i}$ for $i=1,2, \cdots, n$.

(iv) follows immediately from (iii).

COROLlaRY 2.2. If $F$ is a formally real field satisfying $E D$ then $F$ satisfies SAP.

Proof. This is a consequence of Lemma 2.1 (ii), [3, Th. C], and [8, Satz 3.1] (see also [9, Th. 3.1]).

ExAMPLES. (i) If $F$ has a unique ordering then $F$ satisfies ED.

(ii) Let $F=Q((t))$ be the field of formal power series over $Q$. As observed by Elman, Lam, and Prestel [3], the form $\langle t,-2 t\rangle \in W_{t}(F)$ does not represent a totally negative element and consequently cannot be effectively diagonalized. Thus $F$ does not satisfy ED. Since $F$ has only two orderings, $F$ does satisfy SAP. Thus SAP does not imply ED.

However, we do have the following

Proposition 2.3. A formally real field $F$ satisfies SAP if and only 
if for any form $\phi$ over $F$ there exists an effectively diagonalized form $\psi=\left\langle b_{1}, b_{2}, \cdots, b_{n}\right\rangle, n=\operatorname{dim} \phi$, such that $\phi-\psi \in W_{t}(F)$.

Proof. $\quad \Rightarrow$ As in [9, Th. 3.1] we let $Y_{k}=\left\{<\right.$ in $\left.X \mid \sigma_{<}(\phi)=-n+2 k\right\}$, $k=0,1, \cdots, n$. Then the family $\left\{Y_{k} \mid k=0,1, \cdots, n\right\}$ is a partition of $X$ and each $Y_{k}$ is an open and closed subset of $X$. Since $F$ satisfies SAP, there exist elements $b_{1}, b_{2}, \cdots, b_{n+1}$ in $\dot{F}$ such that $W\left(b_{i}\right)=Y_{0} \cup Y_{1}$ $\cup \cdots \cup Y_{i-1}, i=1,2, \cdots, n+1$. Then $W\left(b_{i}\right) \subset W\left(b_{i+1}\right)$ for all $i$ and one readily checks that $\sigma_{<}\left(\left\langle b_{1}, b_{2}, \cdots, b_{n}\right\rangle\right)=\sigma_{<}(\phi)$ for all orderings $<$ in $X$. Hence $\phi-\left\langle b_{1}, b_{2}, \cdots, b_{n}\right\rangle$ lies in $W_{t}\left(F^{\prime}\right)$.

$(\Leftrightarrow)$ By [3, Th. C] and [8, Satz 3.1] it is enough to show that if $\phi$ is totally indefinite then there exists $m \geq 1$ such that $m \phi$ is isotropic. Let $\psi=\left\langle b_{1}, b_{2}, \cdots, b_{n}\right\rangle, n=\operatorname{dim} \phi$, be an effectively diagonalized form with $\phi-\psi \in W_{t}\left(F^{\prime}\right)$. Then there exists an integer $r \geq 1$ such that $r \phi \cong r \psi$. Since $\phi$ is totally indefinite, this implies $\psi$ is also totally indefinite so by Lemma 2.1 (ii) there exists an integer $s \geq 1$ such that $s \psi$ is isotropic. Hence if $m=r s$ then $m \phi$ is isotropic.

THEOREM 2.4. For a formally real field $F$ the following statements are equivalent:

(i) F satisfies ED.

(ii) If $\phi$ is a form over $F$ which represents 1 over all real closures of $F$ then $\phi$ represents a totally positive element of $F$.

Proof. (i) $\Rightarrow$ (ii). Write $\phi=\left\langle a_{1}, a_{2}, \cdots, a_{n}\right\rangle$ with $W\left(a_{i}\right) \subset W\left(a_{i+1}\right)$. Since $\phi$ represents 1 over all real closures it follows that $W\left(a_{1}\right)=\phi$, i.e. $a_{1}$ is totally positive.

(ii) $\Rightarrow$ (i). We first show that any totally indefinite form over $F$ is weakly isotropic and hence, in view of [3,8], $F$ satisfies SAP. If $\phi$ is totally indefinite then $\phi$ represents 1 over all real closures and hence we can write $\phi=\langle a\rangle \perp \phi_{1}$ where $a$ is totally positive element of $F$. But then $\phi_{1}$ represents -1 over all real closures so $\phi_{1}$ represent a totally negative element $b$ in $\dot{F}$. Since $\langle a, b\rangle \in W_{t}(F)$ it follows that $\phi=\langle a, b\rangle \perp \psi$ is weakly isotropic.

Now let $\psi$ be any form over $F$. Since $F$ satisfies SAP there exists $b$ in $\dot{F}$ such that $W(b)=\left\{\langle\epsilon X| \sigma_{<}(\psi)=-\operatorname{dim} \psi\right\}$. If $W(b)$ is empty then $\psi$ represents 1 over all real closures and hence represents a totally positive element. In this case the proof is finished by induction on $\operatorname{dim} \psi$. Hence we can assume that $W(b)$ is non empty. Now $W(b) \subset W(c)$ for 
all elements $c \neq 0$ represented by $\psi$ and $\psi \perp\langle-b\rangle$ represents 1 over all real closures. Thus $\psi \perp\langle-b\rangle$ represents a totally positive element $d$. Since $-b$ is not totally positive we can write $d=a-b x^{2}$ where $a \neq 0$ is represented by $\psi$. Then $W(a) \subset W(b)$ so that $W(a) \subset W(c)$ for all $c$ in $\dot{F}$ represented by $\psi$. Thus induction on $\operatorname{dim} \psi$ completes the proof.

COROLlaRY 2.5. If $F$ is a formally real field satisfying some Hasse Principle $H_{n}$ with $n \geq 4$ then $F$ satisfies $E D$.

Proof. Let $\phi$ be a form over $F$ which represents 1 over all real closure of $F$. Then $\phi \perp n\langle-1\rangle$ is totally indefinite whence isotropic. Thus there exists $x_{1}, \cdots, x_{n}$ in $F$ such that $\phi$ represents the totally positive element $x_{1}^{2}+\cdots+x_{n}^{2} \in \dot{F}$.

COROLlaRY 2.6 (cf. [1, Th. 5.3]). For a formally real pythagorean field $F$ the following statements are equivalent:

(i) $F$ satisfies $S A P$.

(ii) F satisfies $E D$.

(iii) $F$ satisfies $H_{n}$ for all $n \geq 2$.

Proof. The equivalence of (i) and (ii) is a consequence of Proposition 2.3 and the equivalence of (ii) and (iii) follows from Lemma 2.1 (i) and Corollary 2.5.

\section{Hasse principles and the $u$-invariant}

Any non-formally real field vacuously satisfies ED since $X=X(F)$ is empty but need not satisfy $H_{n}$ for any $n$. In fact, for $F$ non-formally real, $F$ satisfies $H_{n}$ for some $n \geq 2$ if and only if $u=u(F)$ is finite. For formally real fields we have

THEOREM 3.1. Let $F$ be a formally real field having at most a finite number of orderings. Then the following statements are equivalent:

(i) $F$ satisfies $H_{n}$ for some $n \geq 4$.

(ii) $F$ satisfies $E D$ and $u(F)<\infty$.

Before proving Theorem 3.1 we introduce some terminology. A quadratic form $\phi$ over $F$ will be called totally positive if every non zero element of $F$ represented by $\phi$ is totally positive. Thus $\phi$ is totally positive if and only if $\phi=\left\langle a_{1}, \cdots, a_{n}\right\rangle$ with $a_{i} \in \Sigma \dot{F}^{2}, i=1, \cdots, n$, if and only if $\sigma_{<}(\phi)=\operatorname{dim} \phi$ for all orderings $<$ of $F$. Denote by $h$ the exponent 
of $W_{t}(F) . \quad h$ is called the height of $F$ and (when finite) $h=2^{m}$ where $m \geq 0$ is the smallest integer such that every totally positive element of $F$ is a sum of $2^{m}$ squares in $F[6$, p. 311]. It follows immediately that if $u(F)$ is finite then $h$ is finite and $h \leq u(F)$.

The proof of Theorem 3.1 will use the following lemma:

LEMMA 3.2. Suppose $F$ is a field with $u=u(F)<\infty$. If $\phi$ is a totally positive form over $F$ with $\operatorname{dim} \phi>4^{m}(u+1)$ for some $m \geq 0$ then there exists a in $\Sigma \dot{F}^{2}$ such that $\phi=2^{m+1}\langle a\rangle \perp \psi$.

Proof. We proceed by induction on $m$. If $m=0$ then $\operatorname{dim} \phi>u+1$ so there exists an integer $n$ with $u+1 \leq 2 n \leq \operatorname{dim} \phi$. Write $\phi=\left\langle a_{1}\right.$, $\left.\cdots, a_{n}, b_{1}, \cdots, b_{n}\right\rangle \perp \phi^{\prime}$. Then $\left\langle a_{1}, \cdots, a_{n},-b_{1}, \cdots,-b_{n}\right\rangle \in W_{t}(F)$ and has dimension larger than $u$. Hence $\left\langle a_{1}, \cdots, a_{n}\right\rangle$ and $\left\langle b_{1}, \cdots, b_{n}\right\rangle$ represent a common element $a \in \Sigma \dot{F}^{2}$. Thus $\phi=2\langle a\rangle \perp \psi$.

Now assume $m>0$ and choose $\phi_{1}$ of biggest dimension such that $\phi=2 \phi_{1} \perp \phi_{2}$. Then the foregoing argument shows that $\operatorname{dim} \phi_{2} \leq u+1$. Hence $\operatorname{dim} \phi_{1}>\frac{1}{2}\left(4^{m}-1\right)(u+1)$. But $m>0$ implies that $\frac{1}{2}\left(4^{m}-1\right)>4^{m-1}$ so $\operatorname{dim} \phi_{1}>4^{m-1}(u+1)$. Hence by the induction hypothesis there exists $a$ in $\Sigma \dot{F}^{2}$ such that $\phi_{1}=2^{m}\langle a\rangle \perp \psi_{1}$. But then $\phi=2^{m+1}\langle a\rangle \perp \psi$ where $\psi=2 \psi_{1} \perp \phi_{2}$.

Proof of Theorem 3.1. (i) $\Rightarrow$ (ii). This follows from Corollary 2.5 and the fact that if $H_{n}$ holds for some $n \geq 2$ then $u(F)<n$. (ii) $\Rightarrow$ (i). Let $s<\infty$ be the number of orderings on $F$. Since $u=u(F)$ is finite the height $h$ of $F$ is also finite (with $h \leq u$ ) so we can write $h=2^{m}$ for some integer $m \geq 0$. We now assert that if $n>(s+1)\left(\frac{h}{2}\right)^{2}$ $\cdot(u+1)$ then $H_{n}$ holds. To see this let $\phi$ be a totally indefinite form over $F$ with $\operatorname{dim} \phi>(s+1)\left(\frac{h}{2}\right)^{2}(u+1)$. Since $F$ satisfies ED we can find elements $a_{i j}$ in $F, 1 \leq i \leq k, 1 \leq j \leq n_{i}$, such that for each $i, W\left(a_{i 1}\right)$ $=\cdots=W\left(a_{i n_{i}}\right), W\left(a_{i 1}\right) \subsetneq W\left(a_{i+1,1}\right)$, and $\phi=\phi_{1} \perp \phi_{2} \perp \cdots \perp \phi_{k}$ where $\phi_{i}=$ $\left\langle a_{i 1}, a_{i 2}, \cdots, a_{i n_{i}}\right\rangle$. Then by choosing orderings in $W\left(a_{i+1,1}\right)-W\left(a_{i 1}\right), i=$ $1,2, \cdots, k-1$ we see that $s \geq k-1$. Hence $\operatorname{dim} \phi=n_{1}+n_{2}+\cdots+n_{k}$ $>(s+1)\left(\frac{h}{2}\right)^{2}(u+1) \geq k\left(\frac{h}{2}\right)^{2}(u+1)$. Thus there must exist some $i$ 
with $n_{i}>\left(\frac{h}{2}\right)^{2}(u+1)=4^{m-1}(u+1)$. Now $W\left(a_{i 1}\right)=\cdots=W\left(a_{i n_{i}}\right)$ so the form $\left\langle a_{i 1}\right\rangle \phi_{i}=\left\langle a_{i 1}\right\rangle \otimes \phi_{i}$ is totally positive and hence by Lemma 3.2, $\left\langle a_{i 1}\right\rangle \phi_{i}$ $=2^{m}\langle a\rangle \perp \psi$ for some $a$ in $\Sigma \dot{F}^{2}$. Hence $\left\langle a_{i 1}\right\rangle \phi=2^{m}\langle a\rangle \perp \phi^{\prime}$ for some subform $\phi^{\prime}$. Let $\phi^{\prime}=\left\langle b_{1}, b_{2}, \cdots, b_{r}\right\rangle$ be an effective diagonalization of $\phi^{\prime}$. Then $\left\langle a_{i 1}\right\rangle \phi=2^{m}\langle a\rangle \perp\left\langle b_{1}, b_{2}, \cdots, b_{r}\right\rangle$ is an effective diagonalization. Since $\phi$ is totally indefinite so is $\left\langle a_{i 1}\right\rangle \phi$ so $b_{r}$ must be totally negative. But $h=2^{m}$ implies that $2^{m}\langle a\rangle$ represents all totally positive elements of $F$. Thus $\left\langle a_{i 1}\right\rangle \phi$ is isotropic whence $\phi$ is also isotropic.

Remark. For many fields the bound $\left(n>(s+1)\left(\frac{h}{2}\right)^{2}(u+1)\right)$ obtained in the proof of Theorem 3.1 is not very precise. In the case that $F=$ $Q$, the proof shows that $H_{n}$ holds for all $n>40$ while it is well known that $n \geq 5$ suffices. Moreover, there exist fields having an infinite number of orderings (for example, the pythagorean closure of $Q$ ) which satisfy the equivalent conditions of the theorem.

COROLlaRY 3.3. Let $F$ be a field having a unique ordering. Then $u\left(F^{\prime}\right)<\infty$ if and only if $F$ satisfies $H_{n}$ for some $n \geq 2$. In this case, $F$ satisfies $H_{n}$ for all $n>\frac{1}{2} h^{2}(u+1)$.

Proof. A field having a unique ordering satisfies ED.

ExAmple. If $F=Q((t))$ then $F$ has exactly two orderings and $u(F)=8$ but as observed in [3], $F$ fails to satisfy $H_{n}$ for any $n \geq 2$.

\section{Kneser's Theorem}

In this section we present two more generalizations (cf. [2, Th. 2.4, Cor. 2.5, and Th. 3.1]) of Kneser's Theorem which states that if $F$ is a non-formally real field and $q=\left|\dot{F} / \dot{F}^{2}\right|$ then $u(F) \leq q$. For this purpose we introduce the following notation. For a form $\phi$ over $F$, let $D(\phi)=$ $\left\{a \in \dot{F} / \dot{F}^{2} \mid a\right.$ is represented by $\left.\phi\right\}$.

LEMMA 4.1. Let $F$ be a field and $\phi$ a totally positive form over $F$. If $D(\phi) \neq \Sigma \dot{F}^{2} / \dot{F}^{2}$ then for any $a$ in $\Sigma \dot{F}^{2}, D(\phi \perp\langle a\rangle) \neq D(\phi)$.

Proof. If $D(\phi \perp\langle a\rangle)=D(\phi)$ then for any integer $n \geq 1, D(\phi \perp n\langle a\rangle)$ $=D(\phi)$. Now if $b \in \Sigma \dot{F}^{2}$ then $a b$ is a sum of $k$ squares in $F$ for some $k \geq 1$ which implies that $b$ is represented by the form $k\langle a\rangle$. Hence 
$b \in D(\phi \perp k\langle a\rangle)=D(\phi)$, contrary to assumption.

THEOREM 4.2. If $F$ is a formally real field satisfying ED then $u(F) \leq\left|\Sigma \dot{F}^{2} / \dot{F}^{2}\right|$.

Proof. Let $t=\left|\Sigma \dot{F}^{2} / \dot{F}^{2}\right|$. It is enough to show that if $\phi \in W_{t}\left(F^{\prime}\right)$ with $\operatorname{dim} \phi \geq t+2$ then $\phi$ is isotropic. Since $F$ is formally real and satisfies ED we can write $\phi=\left\langle a_{1}, \cdots, a_{m}, b_{1}, \cdots, b_{m}\right\rangle$ where $a_{i} \in \Sigma \dot{F}^{2}$, $b_{i} \in-\Sigma \dot{F}^{2}, i=1, \cdots, m$, and $m \geq \frac{t+2}{2}$. Then by Lemma 4.1, $\mid D\left(\left\langle a_{1}, \cdots\right.\right.$, $\left.\left.a_{m}\right\rangle\right) \mid>\frac{t}{2}$ and $\left|D\left(\left\langle-b_{1}, \cdots,-b_{m}\right\rangle\right)\right|>\frac{t}{2}$. Thus there exists $a \in D\left(\left\langle a_{1}\right.\right.$, $\left.\left.\cdots, a_{m}\right\rangle\right) \cap D\left(\left\langle-b_{1}, \cdots,-b_{m}\right\rangle\right)$. But then $-a \in D\left(\left\langle b_{1}, \cdots, b_{m}\right\rangle\right)$, whence $\phi$ is isotropic.

EXAMPLE. The hypothesis that $\boldsymbol{F}$ satisfies ED is needed here since if we let $F_{0}$ be a formally real field having square classes $\{ \pm 1, \pm 2\}$ (such fields exist by $[4, \mathrm{p} .302]$ ) and let $F=F_{0}((t))$ then $u(F)=4$ but $t=\left|\Sigma \dot{F}^{2} / \dot{F}^{2}\right|=2$.

COROLLARY 4.3. Let $F$ be a formally real field satisfying ED. If $q=\left|\dot{F} / \dot{F}^{2}\right|<\infty$ then $u(F) \leq 2^{-s} q$ where $s$ is the number of distinct orderings of $F$.

Proof. Since $F$ satisfies ED, $F$ also satisfies SAP so it follows from (the proof of) Example 4.10 (iii) in [5] that $\left|\dot{F} / \Sigma \dot{F}^{2}\right|=2^{s}$. Hence $q=$ $\left|\dot{F} / \dot{F}^{2}\right|=\left|\dot{F} / \Sigma \dot{F}^{2}\right|\left|\Sigma \dot{F}^{2} / \dot{F}^{2}\right|=2^{s}\left|\Sigma \dot{F}^{2} / \dot{F}^{2}\right|$.

THEOREM 4.4. Let $F$ be a formally real field which satisfies ED and suppose $q<\infty$. Write $q=2^{s} t$ where $t=\left|\Sigma \dot{F}^{2} / \dot{F}^{2}\right|$ and $s$ is the number of orderings on $F$. Then $F$ satisfies $H_{n}$ for all $n>s(t-1)+1$. In particular, $H_{n}$ holds for all $n \geq \frac{q}{2}+1$.

Proof. Let $\phi$ be a totally indefinite form over $F$ and write $\phi=$ $\left\langle a_{11}, \cdots, a_{1 n_{1}}, a_{21}, \cdots, a_{2 n_{2}}, \cdots, a_{k 1}, \cdots, a_{k n_{k}}\right\rangle$ where, for $i=1,2, \cdots, k, W\left(a_{i 1}\right)$ $=\cdots=W\left(a_{i n_{i}}\right)$ and $W\left(a_{i 1}\right) \subseteq W\left(a_{i+1,1}\right)$. Then $n_{1}+n_{2}+\cdots+\dot{n_{k}}=\operatorname{dim} \phi$ and $k \leq s+1$. If $\phi$ is anisotropic then by Lemma 4.1, $n_{1}+n_{k} \leq t$ since otherwise $D\left(\left\langle a_{11}, \cdots, a_{1 n_{1}}\right\rangle\right)$ and $D\left(\left\langle-a_{k 1}, \cdots,-a_{k n_{k}}\right\rangle\right)$ would have an element in common. Moreover, by replacing $\phi$ by $\left\langle a_{i 1}\right\rangle \phi$ and using effective diagonalization (as in the proof of Theorem 3.1) we see that 
$n_{i} \leq t-1$ for $i=2, \cdots, k-1$. Hence $\operatorname{dim} \phi=n_{1}+n_{2}+\cdots+n_{k} \leq t$ $+(k-2)(t-1) \leq t+(s-1)(t-1)=s(t-1)+1$. Thus if $\operatorname{dim} \phi>$ $s(t-1)+1$ then $\phi$ is isotropic. For the last statement, note that $\frac{q}{2}+1$ $=2^{s-1} t+1>s(t-1)+1$.

COROLlary 4.5. Let $F$ be a field having a unique ordering. If $q<\infty$ then $H_{n}$ holds for all $n>\frac{q}{2}$.

CoRollary 4.6. Let $F$ be a formally real field satisfying $E D$. If $F$ has more than one ordering then $H_{n}$ holds for all $n \geq \frac{q}{2}$.

Proof. If $s \geq 2$ then $\frac{q}{2}=2^{s-1} t>s(t-1)+1$.

\section{REFERENCES}

[1] R. Elman and T. Y. Lam, Quadratic forms over formally real fields and pythagorean fields, Amer. J. Math. 94 (1972), 1155-1194.

[2] R. Elman and T. Y. Lam, Quadratic forms and the $u$-invariant. I, Math. Z. 131 (1973), 283-304.

[ 3 ] R. Elman, T. Y. Lam, and A. Prestel, On some Hasse Principles over formally real fields, Math. Z. 134 (1973), 291-301.

[4] H. Gross and H. R. Fischer, Non-real fields $k$ and infinite dimensional $k$-vector spaces, Math. Ann. 159 (1965), 285-308.

[5] M. Knebusch, A. Rosenberg, and R. Ware, Signatures on semilocal rings, J. Algebra 26 (1973), 208-250.

[6] T. Y. Lam, "The Algebraic Theory of Quadratic Forms", W. A. Benjamin, Reading, Massachusetts, 1973.

[ 7 ] A. Pfister, Quadratische Formen in beliebigen Körpern, Invent. Math. 1 (1966), 116-132.

[ 8 ] A. Prestel, Quadratische Semi-Ordnungen und quadratische Formen, Math. Z. 133 (1973), 319-342.

[9] A. Rosenberg and R. Ware, Equivalent topological properties of the space of signatures of a semilocal ring, Publ. Math. Debrecen, to appear. 